\title{
What interventions can improve quality of life or psychosocial factors of individuals with knee osteoarthritis? A systematic review with meta- analysis of primary outcomes from randomised controlled trials
}

\author{
Ronaldo Valdir Briani, ${ }^{1}$ Amanda Schenatto Ferreira, ${ }^{1}$ Marcella Ferraz Pazzinatto, ${ }^{1}$ \\ Evangelos Pappas, ${ }^{2}$ Danilo De Oliveira Silva, ${ }^{3}$ Fábio Mícolis de Azevedo ${ }^{1}$
}

- Additional material is published online only. To view please visit the journal online (http://dx.doi.org/10.1136/ bjsports-2017-098099).

${ }^{1}$ School of Science and Technology, Laboratory of Biomechanics and Motor Control (LABCOM), São Paulo State University, Presidente Prudente, Brazil 2Discipline of Physiotherapy, Faculty of Health Sciences, The University of Sydney, Sydney, New South Wales, Australia ${ }^{3}$ La Trobe Sports and Exercise Medicine Research Centre (LASEM), La Trobe University, School of Allied Health, Melbourne, Victoria, Australia

Correspondence to Ronaldo Valdir Briani, School of Science and Technology, Laboratory of Biomechanics and Motor Control (LABCOM), São Paulo State University, Presidente Prudente 19060900, Brazil;

ronaldobriani@hotmail.com

Accepted 11 February 2018 Published Online First 16 March 2018

\section{ABSTRACT}

Objective To systematically review evidence of primary outcomes from randomised controlled trials (RCTs) examining the effect of treatment strategies on quality of life $(\mathrm{Q} o \mathrm{~L})$ or psychosocial factors in individuals with knee osteoarthritis (OA).

Design Systematic review with meta-analysis.

Data sources Medline, Embase, SPORTDiscus, the Cumulative Index to Nursing and Allied Health Literature (CINAHL) and Web of Science were searched from inception to November 2017.

Eligibility criteria for selecting studies We included RCTs investigating the effect of conservative interventions on QoL or psychosocial factors in individuals with knee OA. Only RCTs considering these outcomes as primary were included.

Results Pooled data supported the use of exercise therapy compared with controls for improving healthrelated and knee-related QoL. There was limited evidence that a combined treatment of yoga, transcutaneous electrical stimulation and ultrasound may be effective in improving QoL. Limited evidence supported the use of cognitive behavioural therapies (with or without being combined with exercise therapy) for improving psychosocial factors such as self-efficacy, depression and psychological distress.

Summary/Conclusion Exercise therapy (with or without being combined with other interventions) seems to be effective in improving health-related and knee-related QoL or psychosocial factors of individuals with knee OA. In addition, evidence supports the use of cognitive behavioural therapies (with or without exercise therapy) for improving psychosocial factors such as self-efficacy, depression and psychological distress in individuals with knee $\mathrm{OA}$.

PROSPERO registration number CRD42016047602.

\section{INTRODUCTION}

Osteoarthritis (OA) is one of the leading causes of musculoskeletal pain and disability worldwide, ${ }^{1}$ with knee OA affecting up to one-third of people aged over 60 years. ${ }^{2}$ Overall, most individuals with knee OA have chronic pain, ${ }^{3}$ which is a multidimensional experience that influences their quality of life (QoL). ${ }^{4}$
Abnormalities on imaging do not account for all symptoms in individuals with knee OA. ${ }^{5}$ Biopsychosocial models suggest that pain is a complex interaction of factors such as structural damage, peripheral and central pain processing mechanisms, culture, sex, and psychosocial factors. ${ }^{6}$ According to the biopsychosocial model, chronic pain is influenced by a variety of physical and psychosocial factors, including individuals' reaction to pain, which is a product of their thoughts and beliefs (cognitions). ${ }^{4}$ Recently, a systematic review (SR) found evidence for a relationship between psychosocial factors and knee OA. ${ }^{4}$ Individuals with knee OA can have psychological impairments related to coping, self-efficacy, somatising, pain catastrophising and helplessness. ${ }^{4}$

The physical and psychosocial impairments in individuals with knee OA have an impact on QoL due to their influence on social interactions, mental functioning and sleep quality. ${ }^{7}$ QoL refers to the physical, psychological and social domains of health that are influenced by a person's experiences, beliefs, expectations and perceptions. ${ }^{8}$ Individuals with knee OA have inferior QoL compared with controls. ${ }^{79}$ Therefore, therapies that improve QoL in individuals with knee OA may mitigate the clinical, economic and social burden of this disorder. ${ }^{7}$ The purpose of this SR was to synthesise evidence of primary outcomes of randomised controlled trials (RCTs) examining the effect of treatment strategies on QoL or psychosocial factors in individuals with knee OA.

\section{METHODS}

This SR has been completed and reported through consultation with the Preferred Reporting Items for Systematic Reviews and Meta-Analyses guidelines for the reporting of SRs and meta-analyses, ${ }^{10}$ and the SR protocol was registered with PROSPERO (trial registration number: CRD42016047602).

\section{Deviations from study protocol}

Initially, we planned to investigate the effect of any intervention (surgical and conservative) on QoL or psychosocial factors. However, due to the vast differences in outcomes, patient recovery, and type and volume of healthcare utilisation between patients with knee pathology who received 
conservative care versus those who opt for surgery, ${ }^{11}$ we limited this SR to evaluating the effect of conservative (non-surgical) interventions to increase statistical homogeneity. In addition, applying the Template for Intervention Description and Replication (TIDieR) checklist and guide to evaluate how well the interventions were described in the RCTs was decided afterwards. This checklist can inform to what extent the description of the interventions applied in the RCTs was complete and any aspects that are missing. Lastly, the authors were initially interested in investigating the effect of interventions in two common knee disorders, knee OA and patellofemoral pain. However, since no studies were found for patellofemoral pain, this SR is limited to knee OA.

\section{Data sources and search strategy}

Electronic searches of Medline via PubMed, Embase via Ovid, SPORTDiscus, the Cumulative Index to Nursing and Allied Health Literature (CINAHL) via Ebsco and Web of Science were performed from database inception to November 2017. An example of the search strategies can be seen in online supplementary appendix 1 . The electronic search was complemented by hand searching the references of the retrieved articles.

\section{Inclusion and exclusion criteria}

We included RCTs that investigated the effect of any conservative intervention on QoL or psychosocial factors (cognitive or behavioural) in individuals with knee OA. Cognitive factors include coping, self-efficacy, somatisation, pain catastrophising and helplessness; behavioural factors include kinesiophobia (pain-related fear of movement) and pain-related fear avoidance. The primary outcome of RCTs provides the basis for the estimation of sample size, and therefore increases the chances that the study is sufficiently powered to find differences in this specific outcome and the intervention has a higher chance of being targeted to the primary outcome. ${ }^{12} 13$ Thus, focusing on the primary outcome of RCTs is highly recommended as an improvement to methodological rigour. ${ }^{12}$ Therefore, only RCTs that have considered these outcomes as their primary outcome were included in our SR. Abstracts, posters, unpublished studies, non-RCTs, RCTs that did not include QoL or psychosocial factors as primary outcome, and RCTs that did not specify what was the primary outcome were excluded.

An outcome was considered to be 'primary' if the RCT described the outcome as a 'primary', 'key' or 'main' outcome in the publication (regardless of whether a power calculation was performed). For studies that did not specify a primary outcome, the outcome used in the power analysis was considered as the primary outcome. If there was no description of which outcome was the primary and a power analysis was not performed, the study was excluded.

Participants were limited to human, given an established diagnosis of knee OA (tibiofemoral and/or patellofemoral joints) according to accepted criteria. ${ }^{14}$ Participants were required to present with usual pain equal or higher than 3 on a 10 -point scale for a duration of at least 6 months and with at least three of the following: (1) radiographically confirmed knee OA (ie, a score of more than 2 on the Kellgren-Lawrence Scale); (2) to be over 50 years of age with physician-diagnosed knee OA; (3) to suffer from morning stiffness that is relieved in less than $30 \mathrm{~min}$; (4) to have crepitus during active motion; and (5) to show bony tenderness or enlargement without joint warmth. ${ }^{14}$ For studies that recruited mixed (symptomatic) populations, we included only those that reported separately the data on individuals with knee OA. The control group was composed of individuals with the same disorder as those included in the treatment group. There were no other restrictions on the comparison group. There was no restriction on sex, age and year of publication. Language was limited to English or Portuguese. In case of insufficient data, all the authors of the studies were contacted via email. If the authors could not provide the missing data or did not reply to the request after three attempts, the study was excluded.

\section{Review process}

A single investigator (RVB) exported all studies identified by the search strategy to EndNote V.X7.5 (Thomson Reuters, Philadelphia), then cross-referenced and deleted duplicates. Titles and abstracts were screened independently by two researchers (RVB and ASF) for eligibility. Full-text articles of potentially relevant abstracts were retrieved for further review. Any discrepancies were resolved during a consensus meeting, and a third reviewer was available (MFP) if needed.

\section{Methodological quality assessment}

The Physiotherapy Evidence Database (PEDro) was used to evaluate the quality of the studies. This is a validated and reliable appraisal tool designed to assess the risk of bias in clinical trials consisting of 11 items. ${ }^{15}$ Based on this scale, studies were rated as high-quality $(\geq 7 / 10)$, moderate-quality (4-6/10) and low-quality $(\leq 3 / 10) .{ }^{16}$ When available, the score was obtained from PEDro database. When not available, two reviewers (RVB and ASF) independently assessed the quality of the included studies. Then, the TIDieR checklist and guide was used to evaluate how well the interventions were described. ${ }^{17}$ The TIDieR checklist and guide was developed to improve the reporting of interventions across different designs of studies, such as trials, case-control and cohort studies. ${ }^{17}$ In order to adapt it to the study's purpose, a template similar to the PEDro scale was created. Therefore, based on this scale, studies were rated as good intervention description $(\geq 9 / 12)$, moderate intervention description (6-8/12) and poor intervention description $(\leq 5 / 12) .{ }^{17} 18$ It is important to acknowledge that this was a customised rating classification since there is currently no rating classification in the literature. For both scales, any discrepancies were resolved during a consensus meeting, and a third reviewer was available (MFP) if needed. We did not evaluate the publication bias domain in this SR as it is not recommended to assess funnel plot asymmetry with a meta-analysis of fewer than 10 trials. $^{19}$

\section{Assessment of risk of bias}

Two authors (RVB and ASF) independently assessed the risk of bias of the included studies using the Cochrane risk of bias tool. ${ }^{19}$ Any disagreements were resolved during a consensus meeting, adjudicated if necessary by a third reviewer (MFP). The following domains were assessed: random sequence generation; allocation concealment; blinding of participants and personnel; blinding of outcome assessment; incomplete outcome data; selective reporting; and other bias. Other sources of bias were imbalances in baseline characteristics; lack of comparability in clinicians' experience with the interventions under test; and compliance with the intervention. The domains were classified as low risk of bias, high risk of bias and unclear risk of bias (where 'unclear' relates to lack of information or uncertainty over the potential for bias). ${ }^{19}$

\section{Data extraction}

Study characteristics including publication details (author and year), participant characteristics (age, sex, body mass index 
(BMI) and number of participants in each group), outcome measure (QoL, self-efficacy, coping strategies, helplessness and pain catastrophising), treatments applied in the intervention and control groups, and a summary of main findings were extracted from each included study. In terms of QoL, health-related (eg, 36-Item Short-Form Health Survey, SF-36) and knee-related (eg, Knee Injury and Osteoarthritis Outcome Score) instruments were considered as outcomes according to recommendations. ${ }^{820}$ Incorporating generic and disease-specific outcome measurements is important to study the health status when evaluating the effect of treatments on QoL. ${ }^{21}$ All outcomes included in this SR have been previously validated (with their psychometric properties tested) as per the validation studies. ${ }^{2-34}$ For meta-analysis of data, means and SD post treatment were sourced from the original papers when available, or by contacting the authors via email when these data were missing.

\section{Data management and statistical analysis}

Data analysis was completed using the Review Manager Software Package RevMan V.5.3. Data were pooled where studies investigated similar interventions (ie, intervention with the same purpose, eg, muscle strengthening) using comparable outcome measures (eg, SF-36 and Assessment of Quality of Life). Data that could not be pooled were summarised in forest plots (without an overall pooled estimate of effect) to allow easy visualisation of the results. We calculated the standardised mean differences (SMD) with 95\% CIs for both pooled and unpooled continuous data from the end of treatment. Meta-analyses were performed using a random-effects model for analyses and pooled point estimate and $95 \%$ CIs were calculated with tests of heterogeneity. The impact of heterogeneity on meta-analysis results was quantified by the $\mathrm{I}^{2}$ statistic. Also, when appropriate, sensitivity analyses were performed considering the effects of risk of bias by excluding trials with high risk of bias. Following methodology proposed by Hume et al, ${ }^{35}$ calculated individual or pooled SMDs were categorised as small $(\leq 0.59)$, medium $(0.60-1.19)$ or large $(\geq 1.20)$. These criteria were chosen to increase rigour compared with traditional criteria. ${ }^{36}$ Levels of evidence for each finding were established based on an updated version of van Tulder's criteria $^{37}$ :

1. strong: provided by pooled results derived from three or more studies, including a minimum of two high-quality studies, which were statistically homogeneous $(\mathrm{P}>0.05)$; may be associated with a statistically significant or non-significant pooled result

2. moderate: provided by statistically significant pooled results derived from multiple studies that were statistically heterogeneous $(\mathrm{P}<0.05)$, including at least one high-quality study; or from multiple low-quality studies, which were statistically homogeneous $(\mathrm{P}>0.05)$

3. limited: provided by results from one high-quality study or multiple low-quality studies that are statistically heterogeneous $(\mathrm{P}<0.05)$

4. very limited: provided by results from one low-quality study

5. conflicting evidence: provided by inconsistent findings among multiple trials and derived from multiple studies regardless of quality that are statistically heterogeneous.

\section{RESULTS}

Search results and a flow diagram of study selection are summarised in figure 1. Initially, 24 studies were eligible for inclusion in this SR. However, one data set was duplicated and published in two different manuscripts ${ }^{38}{ }^{39}$; therefore, the later

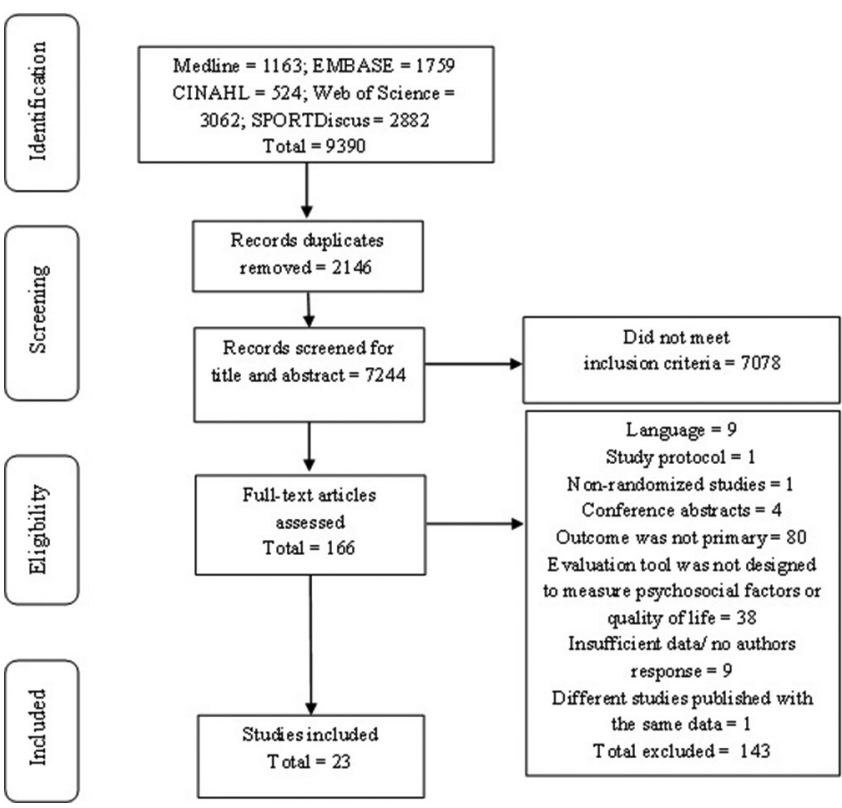

Figure 1 Flow diagram of study selection. Note: the Cumulative Index to Nursing and Allied Health Literature (CINAHL).

one was excluded. ${ }^{38}$ From the 23 studies included in this SR, most studies $(n=19)$ evaluated QoL, ${ }^{39-57}$ one study evaluated self-efficacy ${ }^{56}$ one study evaluated helplessness, ${ }^{58}$ one evaluated pain catastrophising, ${ }^{56}$ two evaluated coping strategies, ${ }^{5659}$ one evaluated depression and psychological distress, ${ }^{60}$ and one evaluated cognitive symptom management $(\mathrm{CSM})^{61}$ in individuals with knee OA. The study of Broderick et $a l^{56}$ considered more than one outcome as primary.

\section{Participants' characteristics of included studies}

Across all studies, there were 3668 participants with knee OA (2607 women: $71.1 \%$ ). The mean age ranged from 56.8 to 67.0 years old and the BMI ranged from 26.4 to 33.7. Of the 3668 participants, $1991(54.3 \%)$ were included in the intervention groups and $1677(45.7 \%)$ were included in the control groups. Details of characteristics of each study are outlined in online supplementary appendix 2 , and the overall characteristics of the excluded studies are presented in online supplementary appendix 3 .

\section{Methodological quality assessment}

The agreements between raters in terms of PEDro scale and TIDieR checklist were $83.3 \%$ and $80.5 \%$, respectively. Quality assessment results are presented in online supplementary appendices 4 and 5. Twelve studies were classified as high quality 41444464849525355565960 and 11 studies were classified as moderate quality. ${ }^{39} 40424548505154575861$ Inadequate blinding of subjects and therapists, and concealed allocation to groups, were the most common methodological limitations. Regarding TIDieR checklist, 11 studies had a good description of the interventions, ${ }^{40} 43-454751535456596011$ studies had a moderate description $39424648-505255575861$ and 1 study had a poor description. ${ }^{41}$ The most common items lacking were (1) describing the intervention provider and their expertise, background and any specific training given; (2) providing information on where physical or informational materials used in the intervention can be accessed; and (3) describing if intervention adherence or fidelity was assessed, how and by whom, and if any strategies were used to maintain or improve fidelity. 


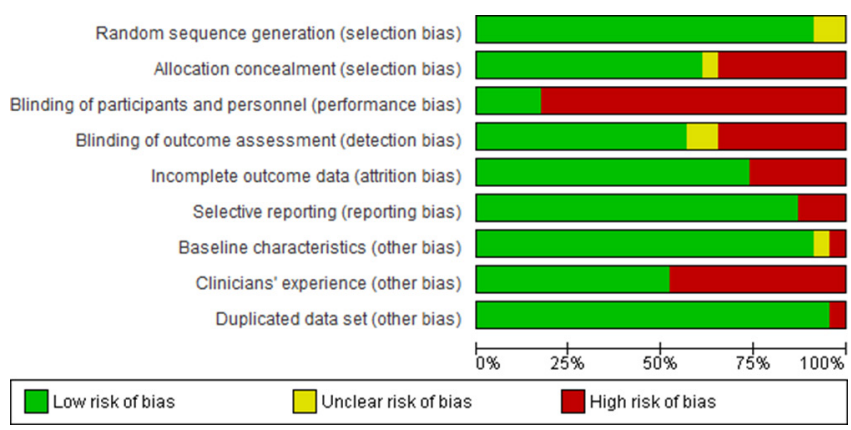

Figure 2 Risk of bias graph: review authors' judgements about each risk of bias item presented as percentages across all included studies.

Risk of bias in included studies

Full descriptions of the risk of bias for the 23 trials are provided in online supplementary appendix 6 and in figures 2 and 3 . Overall, the domains with the highest risk of bias were (1) blinding of participants, (2) clinicians' experience and (3) blinding of outcome assessment.

\section{Effects of interventions}

Meta-analyses could only be performed for QoL due to the low number of studies investigating psychosocial outcomes. A detailed description of each intervention of all studies included in this SR is presented in online supplementary appendix 7.

Effects of interventions on QoL

The results that could be pooled are presented through meta-analyses (figures 4 and 5) and those that could not be pooled are summarised in figure 6 .

\section{Exercise therapy versus control on health-related QoL}

There was moderate evidence with medium effect from four studies (532 participants) favouring exercise therapy to improve QoL of individuals with knee OA compared with no treatment, self-management or anti-inflammatory drugs $(\mathrm{SMD}=0.70$, CI 0.20 to $\left.1.20, \mathrm{P}=0.006, \mathrm{I}^{2}=84 \%\right)^{39424551}$ (figure $4 \mathrm{~A}$ ). Exercise therapy was superior in improving health-related QoL compared with self-management booklets ( $\mathrm{SMD}=0.39$, CI 0.05 to 0.73$)^{51}$ and anti-inflammatory drugs (SMD $=0.47$, CI 0.11 to 0.83$){ }^{45}$ Pooled data from two studies (249 participants) ${ }^{39} 42$ showed that exercise therapy was as effective as no treatment in improving health-related QoL ( $\mathrm{SMD}=1.68$, CI -1.04 to 4.40$)$. However, on removal of Aglamiş et al's study ${ }^{39}$ due to the high risk of bias (presented high risk of bias in six out of nine domains), the results were in favour of exercise therapy in improving health-related QoL compared with no treatment $(\mathrm{SMD}=0.36$, CI 0.11 to 0.36 ), and the high heterogeneity of the overall effect was reduced to $\mathrm{I}^{2}=0 \%$.

\section{Exercise therapy versus control on knee-related QoL}

Overall, there was moderate evidence with small effect from two studies (149 participants) that exercise therapy was superior in improving knee-related QoL compared with no treatment or self-management booklet $(\mathrm{SMD}=0.43$, CI 0.10 to 0.75) (figure 4B). Specifically, exercise therapy was superior in improving knee-related QoL compared with self-management booklet (SMD $=0.59$, CI 0.16 to 1.02$).{ }^{53}$ Exercise therapy was as effective as no treatment $(\mathrm{SMD}=0.21, \mathrm{CI}-0.30$ to 0.71$) .{ }^{54}$

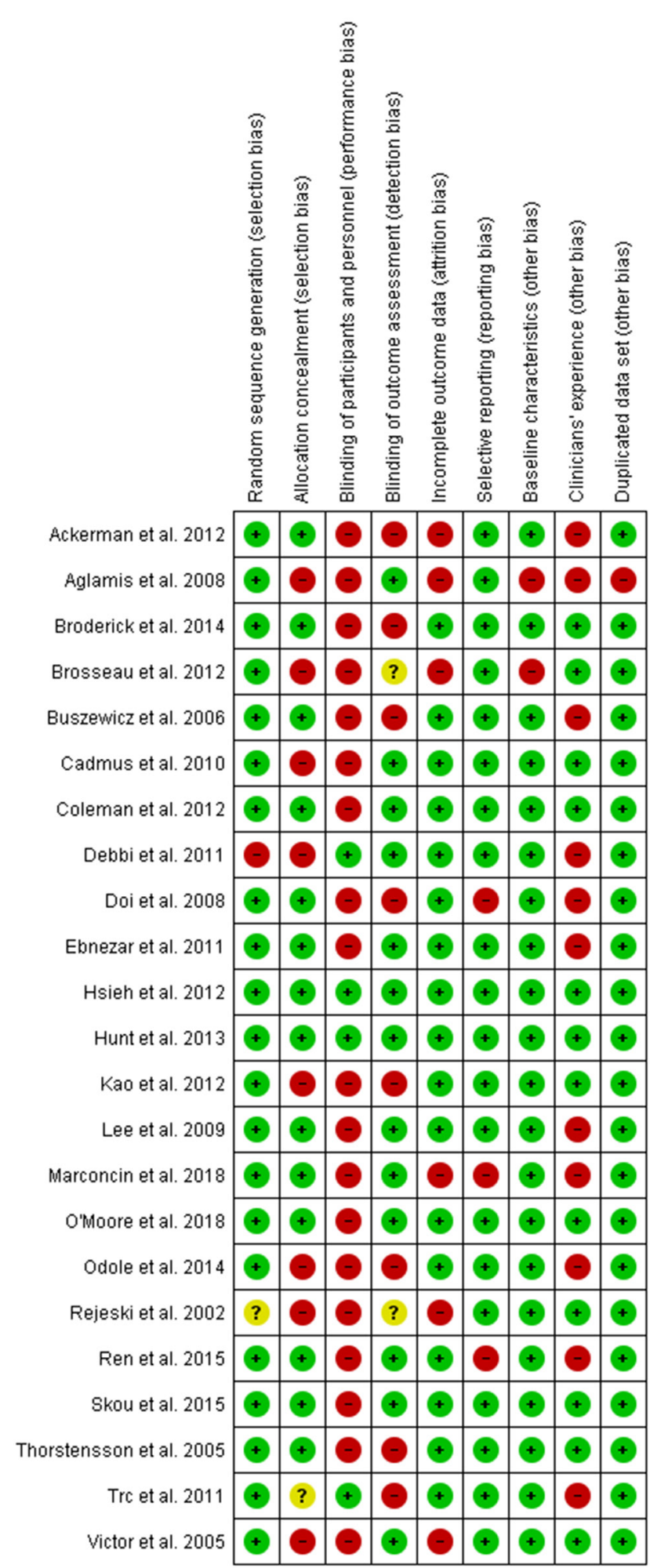

Figure 3 Risk of bias summary: review authors' judgements about each risk of bias item for each included study.

\section{Physical activity plus self-management booklet versus self- management booklet alone}

There was moderate evidence from two studies ${ }^{40} 48$ (395 participants) that physical activity plus self-management booklet was as effective as a self-management booklet alone in improving QoL of individuals with knee $\mathrm{OA}$ ( $\mathrm{SMD}=0.00, \mathrm{CI}-0.20$ to 0.20 , $\mathrm{P}=0.98, \mathrm{I}^{2}=0 \%$ ) (figure $5 \mathrm{~A}$ ). 


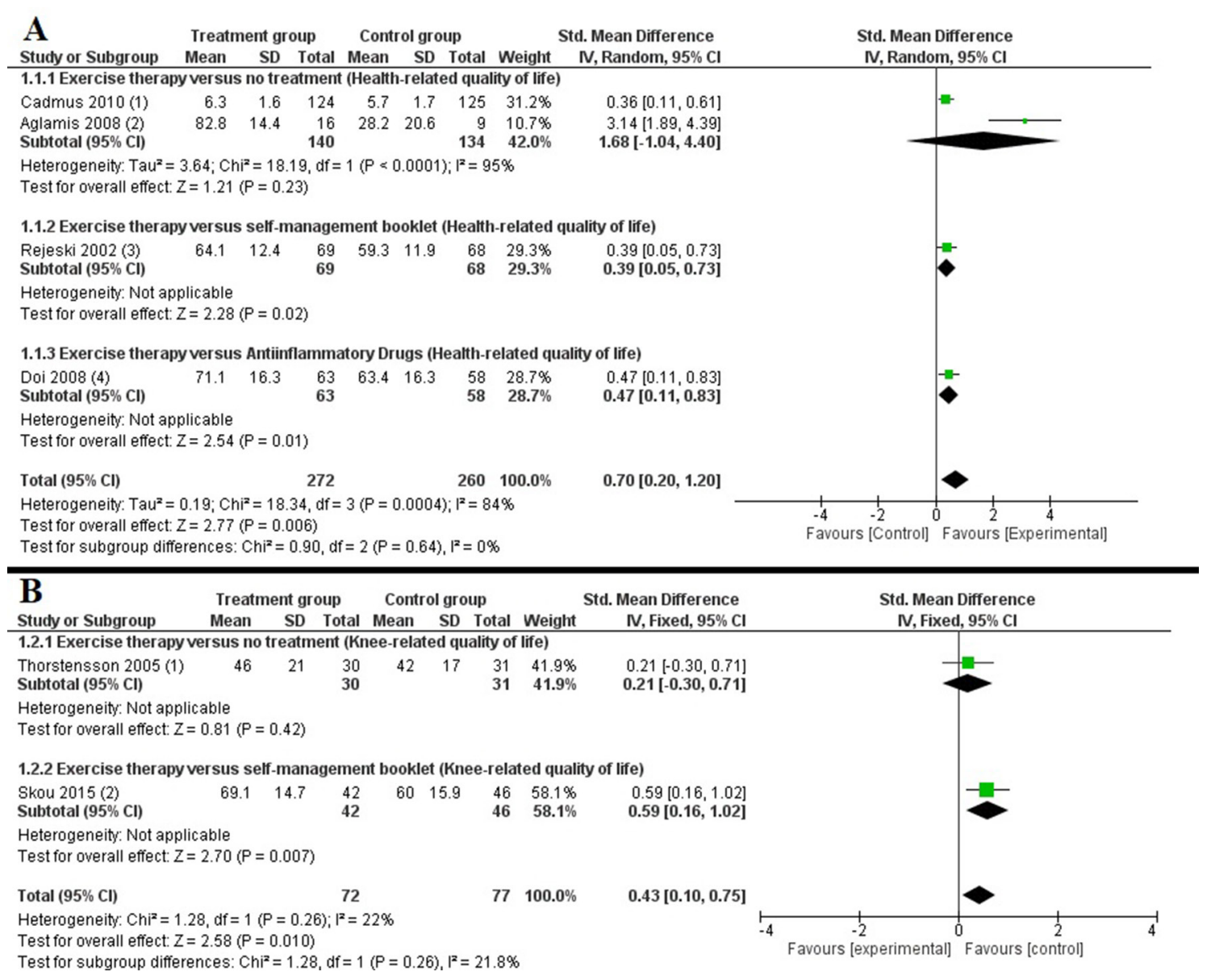

Figure 4 Meta-analyses of exercise therapy on health-related (A) and knee-related (B) QoL. Notes for (A) (time of post-treatment assessment and outcome measure): (1) 20 weeks, PQOL (1-10); (2) 12 weeks, SF-36 (1-100); (3) mixed data from 6 and 18 months, SF-36 (0-100); (4) 11 weeks, SF 36 (1-100). Notes for (B) (time of post-treatment assessment and outcome measure): (1) 6 weeks, KOOS-QoL (0-100); (2) 3 months, KOOS-QoL (1-100). KOOS, Knee Injury and Osteoarthritis Outcome Score; PQOL, Perceived Quality of Life; QoL, quality of life.

\section{Self-management programme (meetings) versus control (no treatment or self-management booklet)}

There was strong evidence from three studies ${ }^{415657}$ (1143 participants) that self-management programmes (meetings) were as effective as a self-management booklet in improving QoL in individuals with knee $\mathrm{OA}(\mathrm{SMD}=0.01, \mathrm{CI}-0.18$ to $0.21, \mathrm{P}=0.89$, $\mathrm{I}^{2}=46 \%$ ) (figure $5 \mathrm{~B}$ ). There was limited evidence from one study (136 participants) ${ }^{43}$ that a self-management programme was as effective as no treatment in improving QoL in individuals with knee $\mathrm{OA}(\mathrm{SMD}=0.33, \mathrm{CI}-0.01$ to $0.67, \mathrm{P}=0.06)$. There was strong evidence from four studies (1279 participants) that self-management programmes (meetings) were as effective as control (self-management booklet or no treatment) in improving QoL of individuals with knee $\mathrm{OA}(\mathrm{SMD}=0.07$, CI -0.12 to $\left.0.26, \mathrm{P}=0.47, \mathrm{I}^{2}=51 \%\right)$.

\section{Effects of other interventions on QoL}

There was limited evidence with medium effect favouring the experimental group that an integrated approach of yoga, transcutaneous electrical stimulation and ultrasound compared with exercise therapy, transcutaneous electrical stimulation and ultrasound $(\mathrm{SMD}=0.65 \text {, CI } 0.40 \text { to } 0.90)^{46}$ was superior in improving QoL in individuals with knee OA (figure 6).

There was limited evidence that (1) moxibustion treatment compared with placebo $(\mathrm{SMD}=0.09 \text {, CI }-0.24 \text { to } 0.43)^{52}$; (2) short-term monochromatic infrared energy compared with placebo $(\mathrm{SMD}=-0.09, \mathrm{CI}-0.55 \text { to } 0.37)^{47}$; (3) methylsulfonylmethane supplementation compared with placebo $(\mathrm{SMD}=0.42$, CI -0.14 to 0.98$)^{44}$; (4) Tai Chi Qigong compared with no treatment $(\mathrm{SMD}=0.46 \text {, CI }-0.17 \text { to } 1.09)^{49}$; and (5) enzymatic hydrolysed collagen compared with glucosamine sulfate $(\mathrm{SMD}=0.12, \mathrm{CI}-0.28 \text { to } 0.53)^{55}$ were as effective in improving QoL of individuals with knee OA (figure 6).

There was very limited evidence with small effect that a telephone-monitored exercise was as effective as clinic-based exercise $(\mathrm{SMD}=0.03 \text {, CI }-0.52 \text { to } 0.59)^{50}$ in improving QoL in individuals with knee OA (figure 6).

\section{Effects of interventions on psychosocial factors}

\section{Effects of interventions on CSM}

There was very limited evidence from one study ${ }^{61}$ with small effect in favour of a combined self-management programme and exercise intervention to improve CSM of individuals with knee OA compared with self-management programme only $(\mathrm{SMD}=0.52$, CI 0.03 to 1.01 ) (figure 6).

\section{Effects of interventions on helplessness}

There was limited evidence from one study ${ }^{58}$ that a care-based, nurse-led education programme was as effective as an education booklet in improving helplessness in individuals with knee OA $(\mathrm{SMD}=-0.20, \mathrm{CI}-0.54$ to 0.14$)$ (figure 6). 


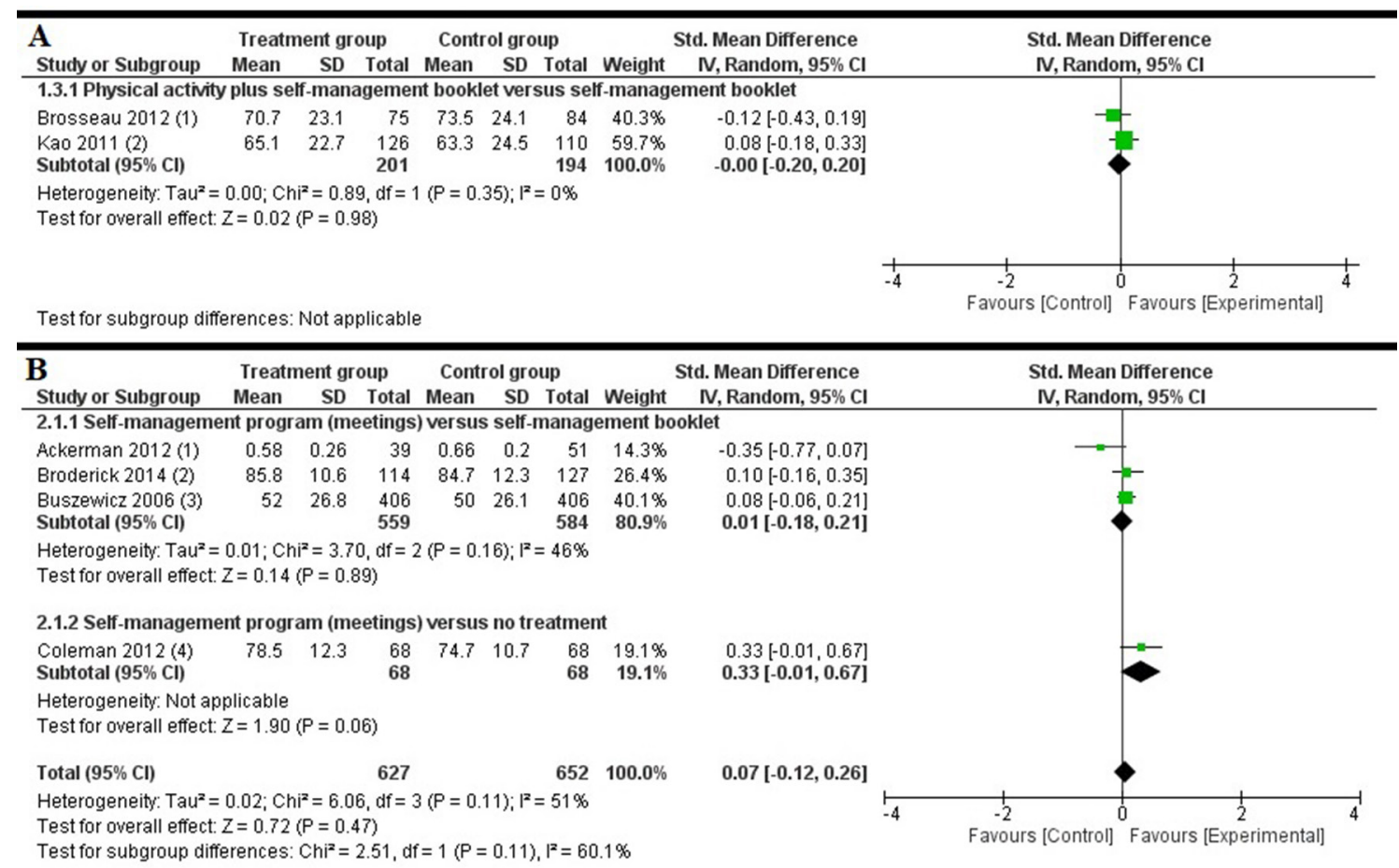

Figure 5 Meta-analyses of physical activity (A) and self-management (B) on health-related QoL. Notes for (A) (time of post-treatment assessment and outcome measure): (1) 12 months, SF-36 (1-100); (2) 4 weeks, SF-36 (1-100). Notes for (B) (time of post-treatment assessment and outcome measure): (1) 3 months, AQoL (-0.04; 1.00); (2) 10-20 weeks from randomisation, QLS (1-100); (3) 4 months, SF-36 (1-100); (4) 8 weeks, SF-36 (1-100). AQoL, Assessment of Quality of Life; QLS, Quality of Life Scale; QOL, quality of life; SF-36, 36-Item Short-Form Health Survey.

\section{Effects of interventions on coping strategies}

Two studies evaluated the effect of interventions on coping strategies. There was limited evidence from two studies ${ }^{56} 59$ that a 10-week physiotherapist-delivered, combined exercise and paincoping skills training intervention was as effective as 10 weeks of exercise integrated with non-directive counselling $(\mathrm{SMD}=0.39$, CI -0.50 to 1.28$),{ }^{59}$ and a coping skills training programme was as effective as usual care $(\mathrm{SMD}=0.24, \mathrm{CI}-0.00 \text { to } 0.49)^{56}$ in improving coping strategies in individuals with knee OA (figure 6).

\section{Effects of interventions on self-efficacy}

There was limited evidence from one study ${ }^{56}$ with medium effect in favour of coping skills training to improve self-efficacy in individuals with knee $\mathrm{OA}$ compared with usual care $(\mathrm{SMD}=0.62$, CI 0.37 to 0.87 ) (figure 6).

\section{Effects of interventions on pain catastrophising}

There was limited evidence from one study ${ }^{56}$ that coping skills training was as effective as usual care in improving pain catastrophising in individuals with knee OA ( $\mathrm{SMD}=0.19$, CI 0.43 to -0.06 ) (figure 6).

\section{Effects of interventions on depression}

There was limited evidence from one study ${ }^{60}$ with medium effect that an internet-based cognitive behaviour therapy on depression improves depression in individuals with knee OA compared with treatment as usual (SMD $=1.01, \mathrm{CI} 1.55$ to 0.47 ) (figure 6).

\section{Effects of interventions on psychological distress}

There was limited evidence from one study ${ }^{60}$ with medium effect that internet-based cognitive behaviour therapy improved psychological distress in individuals with knee OA compared with treatment as usual (SMD $=0.75, \mathrm{CI} 1.28$ to 0.23 ) (figure 6).

\section{DISCUSSION}

This SR identified 23 RCTs that considered QoL or psychosocial factors as primary outcomes. ${ }^{39-61}$ Exercise therapy was more effective than control (no treatment, self-management booklet or anti-inflammatory drugs) in improving QoL in individuals with knee OA. Individual data demonstrated limited evidence that a combined treatment of yoga, transcutaneous electrical stimulation and ultrasound ${ }^{46}$ may be effective in improving QoL of individuals with knee OA. Limited evidence supports the use of cognitive behavioural therapies (with or without being combined with exercise therapy) for improving psychosocial factors such as self-efficacy, depression and psychological distress. ${ }^{5660}$

Our findings support the last Osteoarthritis Research Society International (OARSI) guidelines for the non-surgical management of knee $\mathrm{OA}^{62}$ which recommend exercise therapy (land-based and/or water-based) for improving pain ${ }^{6364}$ and function. ${ }^{65}$ We found that exercise therapy also improved health-related and knee-related QoL. The OARSI recommendations are based on four meta-analyses, ${ }^{63-66}$ in which the duration and type of exercise programmes varied widely, but interventions included a combination of elements such as strength training, active range of motion exercises and aerobic activity. ${ }^{62}$ Similarly, studies from this SR reported that hip and quadriceps strength training, walking programmes, functional exercises, endurance and balance training improved health-related and knee-related QoL. Additionally, a recent study provided an overview of Cochrane reviews to determine the effectiveness of different exercise interventions on 


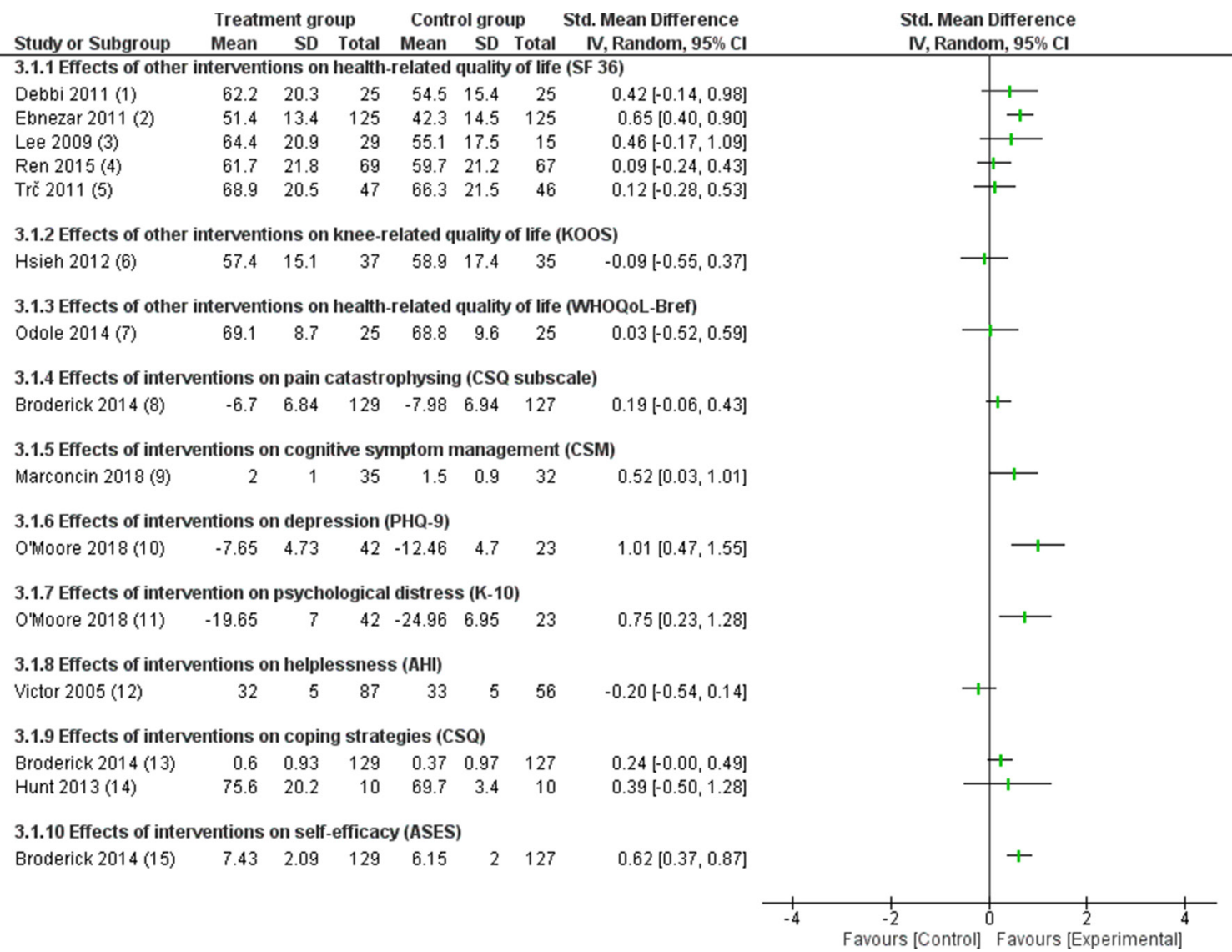

Figure 6 Effects of other interventions on QoL and psychosocial outcomes. Notes (interventions and time of post-treatment assessment): (1) Methylsulfonylmethane supplementation versus placebo, 12 weeks; (2) yoga therapy versus exercise therapy, 2 weeks; (3) Tai Chi Qigong training versus no treatment, 8 weeks; (4) moxibustion treatment versus placebo, 6 weeks; (5) enzymatic hydrolysed collagen versus glucosamine sulfate, 2 weeks; (6) monochromatic infrared energy versus placebo, 2 weeks; (7) telephone-monitored exercise versus clinic-based exercise, 6 weeks; (8) paincoping skills training versus informational brochure, 10-20 weeks; (9) self-management programme and exercise therapy versus self-management, 12 weeks; (10) internet-based cognitive behaviour therapy versus treatment as usual, 11 weeks; (11) internet-based cognitive behaviour therapy versus treatment as usual, 11 weeks; (12) nurse-led education programme versus educational booklet, 1 month; (13) pain-coping skills training versus informational brochure, 10-20 weeks; (14) combined exercise and pain-coping skills versus exercise therapy, 10 weeks; (15) pain-coping skills training versus informational brochure, 10-20 weeks. Mean values of studies 8,10 and 11 were converted to negative values for presentation purposes. KOOS, Knee injury and Osteoarthritis Outcome Score; QoL, quality of life; CSQ, Coping Strategies Questionnaire; ASES, Arthritis Self-Efficacy Scale; AHI, Arthritis Helplessness Index; K-10, Kessler-10; PHQ-9, Patient Health Questionnaire-9; WHOQoL-Bref, World Health Organisation Quality of Life-Bref; SF-36, 36-Item Short-Form Health Survey.

pain, physical function and QoL in adults with chronic pain. ${ }^{67}$ Twenty-one Cochrane reviews were included, and the available evidence suggests that exercise therapy is an intervention with few adverse events that improves pain severity and physical function, and subsequently QoL. ${ }^{67}$ Our findings extend these findings, demonstrating that exercise therapy has positive effects on health-related and knee-related QoL in individuals with knee OA when compared with no treatment, education and anti-inflammatory drugs.

Self-management programmes (meetings) did not improve health-related QoL when compared with a self-management booklet. Despite recommending self-management as appropriate for reducing pain in individuals with knee OA, OARSI guidelines ${ }^{62}$ express reservations about the efficacy and practicality of such interventions. Findings from our SR do not support the recommendation of self-management alone to improve QoL in individuals with knee OA. However, caution in interpreting this finding should be exercised due to the lack of studies considering QoL as a primary outcome.
Adding physical activity to self-management education (booklet) did not improve health-related QoL when compared with self-management booklet only. The intensity of the physical activity performed in these studies was lower than in those entered in the exercise therapy meta-analysis, which may explain the conflicting findings. ${ }^{68}$ A recent SR investigated the benefits and harms of high-intensity versus low-intensity physical activity or exercise programmes in individuals with knee OA. ${ }^{68}$ No conclusive evidence could be drawn due to the small number of studies. However, individuals with knee OA who perform high-intensity exercise may experience slight improvements in knee pain and function at the end of the exercise programme when compared with a low-intensity exercise programme. ${ }^{68}$ Regarding QoL, the high-intensity exercise led to small but superior improvements in QoL. ${ }^{69}$ However, caution in interpreting this finding should be taken since only one study investigated this outcome. 
Yoga combined with transcutaneous electrical stimulation and ultrasound, compared with exercise therapy, transcutaneous electrical stimulation and ultrasound, improved QoL in individuals with knee OA. ${ }^{46}$ Mind-body exercises are promising approaches to reduce pain and improve physical function in individuals with knee OA, ${ }^{70}$ although conflicting findings were reported for QoL. ${ }^{71}$ Similar to our findings, two reviews reported that yoga interventions have positive effects on QoL. ${ }^{70} 71$ However, more RCTs with adequate sample sizes should be conducted before stronger recommendations can be made. ${ }^{70} 71$

In terms of psychosocial outcomes, there was a beneficial effect of pain-coping skills training (10 individual weekly sessions at the doctor's office $)^{56}$ on self-efficacy. An internet-based cognitive behavioural therapy (six online lessons) was also effective in improving depression and psychological distress, ${ }^{60}$ which may negate the necessity of meetings at the clinician's office for educational interventions. For many, accessing specialist clinicians to prescribe and supervise these treatments may be difficult due to cost, transportation issues or geographical location. In this context, a recent RCT found that, for individuals with chronic knee pain, an internet-delivered, physiotherapist-prescribed exercise and pain-coping skills training provides clinically meaningful improvements in pain and function that are sustained for at least 6 months. ${ }^{72}$ Therefore, internet-delivered treatment may be also effective in improving psychosocial outcomes, although more research is needed in this emerging area of research.

\section{Limitations}

There are limitations that need to be considered when interpreting the findings of this SR. We included only RCTs that considered QoL or psychosocial factors as primary outcome to enhance methodological rigour. However, some effective interventions to psychosocial factors or QoL could be missed in RCTs that evaluated psychosocial factors or QoL as secondary outcomes. Publications in languages other than English or Portuguese were not included in our SR, meaning there is a risk for publication bias. The vast majority of studies in other languages tested a pharmaceutical intervention on knee OA, and based on the abstract most did not meet the inclusion criteria. Care should be taken in interpreting the

\section{What is already known on this subject?}

Knee osteoarthritis (OA) is highly prevalent and challenging to manage.

- Individuals with knee OA frequently report poor quality of life (QoL).

- Individuals with knee OA frequently report impaired psychosocial function.

- Interventions that can improve QoL and psychosocial factors are fundamental to managing knee $\mathrm{OA}$.

\section{What are the findings?}

- Exercise therapy improves health-related and knee-related QoL in individuals with knee $O A$.

- Self-management meetings are as effective as booklets in improving QoL in individuals with knee OA.

- Limited evidence supports the use of cognitive behavioural therapies (with or without exercise therapy) for improving psychosocial factors in individuals with knee $O A$. results of this SR since blinding of personnel and outcome assessment was rated as high risk of bias in several studies. Due to the nature of the interventions (eg, exercise therapy compared with no treatment), blinding of personnel and outcome assessment was impractical in many studies.

\section{Future directions}

Despite the evidence presented in this SR, there is a need for further studies investigating the effects of interventions on psychosocial factors in individuals with knee OA. In addition, our findings indicate that the description of the interventions performed in RCTs must be improved as clinicians may not be able to replicate the applied interventions in a clinical environment and researchers cannot replicate them in further RCTs. For instance, from the studies of exercise therapy included in the meta-analyses, two studies ${ }^{39}{ }^{42}$ scored less than 8 (moderate description) in the TIDieR checklist. This makes the findings of the studies difficult to replicate as clinicians are not able to reproduce the interventions in a clinical environment. Also, half of the studies included in our SR were classified as moderate quality in the PEDro checklist, suggesting much of the published data could be biased.

\section{CONCLUSION}

Exercise therapy may be an effective intervention to improve health-related and knee-related QoL in individuals with knee OA. Limited evidence supported the use of exercise therapy alongside self-management programmes to improve psychosocial factors such as CSM. There was limited evidence that combined yoga, transcutaneous electrical stimulation and ultrasound may improve QoL in individuals with knee OA; however, further research is still needed. Limited evidence supported the use of cognitive behavioural therapies (with or without exercise therapy) for improving psychosocial factors such as self-efficacy, depression and psychological distress.

Contributors RVB and ASF were responsible for the systematic search, data extraction and methodological quality appraisals. All authors were involved in the study design, drafting and manuscript editing.

Funding This research did not receive any specific grant from funding agencies in the public, commercial or not-for-profit sectors. RVB, ASF, MFP, and DOS are funded by São Paulo Research Foundation (FAPESP) with scholarships (numbers: 2017/4837, 2016/02357-1, 2016/19784-0, 2015/17777-3). FMA is funded by FAPESP with a grant (number: 2014/24939-7).

Competing interests None declared.

Patient consent Not required.

Provenance and peer review Not commissioned; externally peer reviewed.

(c) Article author(s) (or their employer(s) unless otherwise stated in the text of the article) 2018. All rights reserved. No commercial use is permitted unless otherwise expressly granted.

\section{REFERENCES}

1 Abajobir AA, Abate $\mathrm{KH}$, Abbafati C, et al. Global, regional, and national incidence, prevalence, and years lived with disability for 328 diseases and injuries for 195 countries, 1990-2016: a systematic analysis for the Global Burden of Disease Study 2016. Lancet 2017;390:1211-59.

2 Dillon CF, Rasch EK, Gu Q, et al. Prevalence of knee osteoarthritis in the United States: arthritis data from the third national health and nutrition examination survey 1991 94. J Rheumatol 2006;33:2271-9.

3 Leyland KM, Hart DJ, Javaid MK, et al. The natural history of radiographic knee osteoarthritis: a fourteen-year population-based cohort study. Arthritis Rheum 2012;64:2243-51

4 Urquhart DM, Phyomaung PP, Dubowitz J, et al. Are cognitive and behavioural factors associated with knee pain? a systematic review. Semin Arthritis Rheum 2015:44:445-55 
5 Finan PH, Buenaver LF, Bounds SC, et al. Discordance between pain and radiographic severity in knee osteoarthritis: findings from quantitative sensory testing of central sensitization. Arthritis Rheum 2013;65:363-72.

6 Leeuw M, Goossens ME, Linton SJ, et al. The fear-avoidance model of musculoskeletal pain: current state of scientific evidence. J Behav Med 2007;30:77-94.

7 Farr li J, Miller LE, Block JE. Quality of life in patients with knee osteoarthritis: a commentary on nonsurgical and surgical treatments. Open Orthop J 2013;7:619-23.

8 Testa MA, Simonson DC. Assessment of quality-of-life outcomes. N Eng/ J Med 1996:334:835-40

9 Hoogeboom TJ, den Broeder AA, de Bie RA, et al. Longitudinal impact of joint pain comorbidity on quality of life and activity levels in knee osteoarthritis: data from the Osteoarthritis Initiative. Rheumatology 2013;52:543-6.

10 Liberati A, Altman DG, Tetzlaff J, et al. The PRISMA statement for reporting systematic reviews and meta-analyses of studies that evaluate healthcare interventions: explanation and elaboration. BMJ 2009;339:b2700.

11 Hunter DJ, Lo GH, Gh L. The management of osteoarthritis: an overview and call to appropriate conservative treatment. Rheum Dis Clin North Am 2008;34:689-712.

12 Andrade C. The primary outcome measure and its importance in clinical trials. J Clin Psychiatry 2015;76:e1320-e1323.

13 Stanley K. Design of randomized controlled trials. Circulation 2007:115:1164-9.

14 Altman R, Asch E, Bloch D, et al. Development of criteria for the classification and reporting of osteoarthritis: Classification of osteoarthritis of the knee. Arthritis \& Rheumatism 1986:29:1039-49.

15 Foley NC, Bhogal SK, Teasell RW, et al. Estimates of quality and reliability with the physiotherapy evidence-based database scale to assess the methodology of randomized controlled trials of pharmacological and nonpharmacological interventions. Phys Ther 2006:86:817-24.

16 de Morton NA. The PEDro scale is a valid measure of the methodological quality of clinical trials: a demographic study. Aust J Physiother 2009;55:129-33.

17 Hoffmann TC, Glasziou PP, Boutron I, et al. Better reporting of interventions: template for intervention description and replication (TIDieR) checklist and guide. BMJ 2014;348:g1687.

18 Yamato T, Maher C, Saragiotto B, et al. The TIDieR checklist will benefit the physical therapy profession. J Orthop Sport Phys Ther 2016;46:402-4.

19 Higgins J, Green S. Cochrane Handbook for Systematic Reviews of Interventions Version 5.1.0: Cochrane Collab, 2011.

20 Bellamy N, Kirwan J, Boers M, et al. Recommendations for a core set of outcome measures for future phase III clinical trials in knee, hip, and hand osteoarthritis. Consensus development at OMERACT III. J Rheumatol 1997;24:799-802.

21 Salaffi F, Carotti M, Grassi W. Health-related quality of life in patients with hip or knee osteoarthritis: comparison of generic and disease-specific instruments. Clin Rheumatol 2005:24:29-37.

22 Brady TJ. Measures of self-efficacy, helplessness, mastery, and control. Arthritis Rheum 2003;49:S147-64.

23 Burckhardt CS, Woods SL, Schultz AA, et al. Quality of life of adults with chronic illness: a psychometric study. Res Nurs Health 1989;12:347-54.

24 Hawker GA, Mian S, Kendzerska T, et al. Measures of adult pain. Arthritis Care Res 2011;63:240-52.

25 Jensen MP, Keefe FJ, Lefebvre JC, et al. One and two-item measures of pain beliefs and coping strategies. Pain 2003;104:453-69.

26 Keefe FJ, Caldwell DS, Queen KT, et al. Pain coping strategies in osteoarthritis patients. J Consult Clin Psychol 1987;55:208-12.

27 Lorig K, Chastain RL, Ung E, et al. Development and evaluation of a scale to measure perceived self-efficacy in people with arthritis. Arthritis Rheum 1989;32:37-44.

28 McHorney CA, Ware JE, Raczek AE. The MOS 36-Item Short-Form Health Survey (SF-36): II. Psychometric and clinical tests of validity in measuring physical and mental health constructs. Med Care 1993;31:247-63.

29 Noerholm V, Groenvold M, Watt T, et al. Quality of life in the Danish general population--normative data and validity of WHOQOL-BREF using Rasch and item response theory models. Qual Life Res 2004;13:531-40.

30 Obradovic M, Lal A, Liedgens H. Validity and responsiveness of EuroQol-5 dimension (EQ-5D) versus Short Form-6 dimension (SF-6D) questionnaire in chronic pain. Health Qual Life Outcomes 2013;11:110.

31 Patrick DL, Kinne S, Engelberg RA, et al. Functional status and perceived quality of life in adults with and without chronic conditions. J Clin Epidemiol 2000;53:779-85.

32 Ren XS, Kazis L, Meenan RF. Short-form Arthritis Impact Measurement Scales 2: tests of reliability and validity among patients with osteoarthritis. Arthritis Care Res 1999;12:163-71.

33 Roos EM, Toksvig-Larsen S. Knee injury and Osteoarthritis Outcome Score (KOOS) validation and comparison to the WOMAC in total knee replacement. Health Qual Life Outcomes 2003;1:17.

34 Whitfield K, Buchbinder R, Segal L, et al. Parsimonious and efficient assessment of healthrelated quality of life in osteoarthritis research: validation of the Assessment of Quality of Life (AQoL) instrument. Health Qual Life Outcomes 2006;4:19.

35 Hume P, Hopkins W, Rome K, et al. Effectiveness of foot orthoses for treatment and prevention of lower limb injuries : a review. Sports Med 2008;38:759-79.

36 Cohen J. A power primer. Psychol Bull 1992;112:155-9.
37 van Tulder M, Furlan A, Bombardier C, et al. Updated method guidelines for systematic reviews in the cochrane collaboration back review group. Spine 2003:28:1290-9.

38 Aglamiş B, Toraman NF, Yaman H. Change of quality of life due to exercise training in knee osteoarthritis: SF-36 and WOMAC. J Back Musculoskelet Rehabil 2009;22:43-8

39 Ağlamıs B, Toraman N, Yaman H. The effect of a 12-week supervised multicomponent exercise program on knee OA in Turkish women. J Back Musculoskelet Rehabil 2008:21:121-8

40 Brosseau L, Wells GA, Kenny GP, et al. The implementation of a community-based aerobic walking program for mild to moderate knee osteoarthritis: A knowledge translation randomized controlled trial: Part II: Clinical outcomes. BMC Public Health 2012;12:871.

41 Buszewicz M, Rait G, Griffin M, et al. Self management of arthritis in primary care: randomised controlled trial. BMJ 2006;333:879.

42 Cadmus L, Patrick MB, Maciejewski ML, et al. Community-based aquatic exercise and quality of life in persons with osteoarthritis. Med Sci Sports Exerc 2010:42:8-15

43 Coleman S, Briffa NK, Carroll G, et al. A randomised controlled trial of a selfmanagement education program for osteoarthritis of the knee delivered by health care professionals. Arthritis Res Ther 2012;14:R21.

44 Debbi EM, Agar G, Fichman G, et al. Efficacy of methylsulfonylmethane supplementation on osteoarthritis of the knee: a randomized controlled study. BMC Complement Altern Med 2011;11:50.

45 Doi T, Akai M, Fujino K, et al. Effect of home exercise of quadriceps on knee osteoarthritis compared with nonsteroidal antiinflammatory drugs: a randomized controlled trial. Am J Phys Med Rehabil 2008;87:258-69.

46 Ebnezar J, Nagarathna R, Bali Y, et al. Effect of an integrated approach of yoga therapy on quality of life in osteoarthritis of the knee joint: A randomized control study. Int J Yoga 2011;4:55-63.

47 Hsieh RL, Lo MT, Lee WC, et al. Therapeutic effects of short-term monochromatic infrared energy therapy on patients with knee osteoarthritis: a double-blind, randomized, placebo-controlled study. J Orthop Sports Phys Ther 2012:42:947-56

48 Kao MJ, Wu MP, Tsai MW, et al. The effectiveness of a self-management program on quality of life for knee osteoarthritis (OA) patients. Arch Gerontol Geriatr 2012; $54: 317-24$

49 Lee HJ, Park HJ, Chae Y, et al. Tai Chi Qigong for the quality of life of patients with knee osteoarthritis: a pilot, randomized, waiting list controlled trial. Clin Rehabil 2009:23:504-11.

50 Odole AC, Ojo OD. Is telephysiotherapy an option for improved quality of life in patients with osteoarthritis of the knee? Int J Telemed App/ 2014;2014:1-9.

51 Rejeski WJ, Focht BC, Messier SP, et al. Obese, older adults with knee osteoarthritis: Weight loss, exercise, and quality of life. Health Psychology 2002:21:419-26.

52 Ren X, Yao C, Wu F, et al. Effectiveness of moxibustion treatment in quality of life in patients with knee osteoarthritis: a randomized, double-blinded, placebo-controlled trial. Evid Based Complement Alternat Med 2015;2015:1-6.

53 Skou ST, Rasmussen S, Laursen MB, et al. The efficacy of 12 weeks non-surgical treatment for patients not eligible for total knee replacement: a randomized controlled trial with 1-year follow-up. Osteoarthritis Cartilage 2015;23:1465-75.

54 Thorstensson CA, Roos EM, Petersson IF, et al. Six-week high-intensity exercise program for middle-aged patients with knee osteoarthritis: a randomized controlled trial [ISRCTN20244858]. BMC Musculoskelet Disord 2005;6:27.

55 Trč T, Bohmová J. Efficacy and tolerance of enzymatic hydrolysed collagen (EHC) vs. glucosamine sulphate (GS) in the treatment of knee osteoarthritis (KOA). Int Orthop 2011:35:341-8.

56 Broderick JE, Keefe FJ, Bruckenthal P, et al. Nurse practitioners can effectively deliver pain coping skills training to osteoarthritis patients with chronic pain: A randomized controlled trial. Pain 2014;155:1743-54

57 Ackerman IN, Buchbinder R, Osborne RH. Challenges in evaluating an Arthritis Self-Management Program for people with hip and knee osteoarthritis in real-world clinical settings. J Rheumatol 2012;39:1047-55.

58 Victor CR, Triggs E, Ross F, et al. Lack of benefit of a primary care-based nurse-led education programme for people with osteoarthritis of the knee. Clin Rheumatol 2005:24:358-64

59 Hunt MA, Keefe FJ, Bryant C, et al. A physiotherapist-delivered, combined exercise and pain coping skills training intervention for individuals with knee osteoarthritis: a pilot study. Knee 2013;20:106-12.

60 O'moore KA, Newby JM, Andrews G, et al. Internet Cognitive-Behavioral Therapy for Depression in Older Adults With Knee Osteoarthritis: A Randomized Controlled Trial. Arthritis Care Res 2018:70:61-70.

61 Marconcin P, Espanha M, Teles J, et al. A randomized controlled trial of a combined self-management and exercise intervention for elderly people with osteoarthritis of the knee: the PLE²NO program. Clin Rehabil 2018:32:223-32.

62 McAlindon TE, Bannuru RR, Sullivan MC, et al. OARSI guidelines for the non-surgical management of knee osteoarthritis. Osteoarthritis Cartilage 2014;22:363-88. 
63 Fransen M, McConnell S, Harmer AR, et al. Exercise for osteoarthritis of the knee: a Cochrane systematic review. Br I Sports Med 2015;49:1554-7.

64 Iversen MD. Rehabilitation Interventions for Pain and Disability in Osteoarthritis. Orthopaedic Nursing 2012;31:103-8.

65 Jansen MJ, Viechtbauer W, Lenssen AF, et al. Strength training alone, exercise therapy alone, and exercise therapy with passive manual mobilisation each reduce pain and disability in people with knee osteoarthritis: a systematic review. J Physiother 2011;57:11-20.

66 Fransen M, McConnell S, Hernandez-Molina G, et al. Does land-based exercise reduce pain and disability associated with hip osteoarthritis? A meta-analysis of randomized controlled trials. Osteoarthritis Cartilage 2010;18:613-20.

67 Geneen L, Moore RA, Clarke C, et al. Physical activity and exercise for chronic pain in adults: an overview of Cochrane Reviews. Cochrane Database Syst Rev 2017;1:CD011279.

68 Regnaux J, Lefevre-colau M, Trinquart L, et al. High-intensity versus low-intensity physical activity or exercise in people with hip or knee osteoarthritis (Review). Cochrane Database Syst Rev 2015;29:CD010203.
69 McCarthy CJ, Mills PM, Pullen R, et al. Supplementation of a home-based exercise programme with a class-based programme for people with osteoarthritis of the knees: a randomised controlled trial and health economic analysis. Health Technol Assess 2004;8:1-61.

70 Brosseau L, Taki J, Desjardins B, et al. The Ottawa panel clinical practice guidelines for the management of knee osteoarthritis. Part three: aerobic exercise programs. Clin Rehabil 2017;31:612-24.

71 Kan L, Zhang J, Yang Y, et al. The effects of yoga on pain, mobility, and quality of life in patients with knee osteoarthritis: a systematic review. Evid Based Complement Alternat Med 2016;2016:1-10.

72 Bennell KL, Nelligan R, Dobson F, et al. Effectiveness of an internet-delivered exercise and pain-coping skills training intervention for persons with chronic knee pain: a randomized trial. Ann Intern Med 2017;166:453-62. 